DOI $10.7603 / \mathrm{s} 40956-016-0007-\mathrm{z}$

Moroccan J. Pure and Appl. Anal.(MJPAA)

RESEARCH ARTICLE

Volume 2(2), 2016, Pages 65-78

ISSN: $2351-8227$

\title{
Bounds for the weighted Dragomir-Fedotov functional
}

\author{
Mohammad W. Alomari ${ }^{a}$
}

AbStRACT. In literature the Dragomir-Fedotov functional is well known as

$$
\mathcal{D}(f ; u):=\int_{a}^{b} f(x) d u(x)-\frac{u(b)-u(a)}{b-a} \int_{a}^{b} f(t) d t .
$$

In this work a generalization of $\mathcal{D}(f ; u)$ is established. Namely, we define the weighted DragomirFedotov functional such as:

$$
\mathcal{O D}(f, g ; u):=\frac{1}{u(b)-u(a)} \cdot \int_{a}^{b} f(x) d u(x)-\frac{1}{\int_{a}^{b} g(t) d t} \cdot \int_{a}^{b} f(t) g(t) d t,
$$

and hence several bounds are proved.

2010 Mathematics Subject Classification. 26D15, 26D20.

Key words and phrases. Grüss inequality, Riemann-Stieltjes integral, functional.

\section{Introduction}

In order to approximate the Stieltjes integral $\int_{a}^{b} f(x) d u(x)$ by the Riemann integral $\int_{a}^{b} f(t) d t$, Dragomir and Fedotov [13], have established the following functional:

$$
\mathcal{D}(f ; u):=\int_{a}^{b} f(x) d u(x)-\frac{u(b)-u(a)}{b-a} \int_{a}^{b} f(t) d t
$$

provided that the Stieltjes integral $\int_{a}^{b} f(x) d u(x)$ and the Riemann integral $\int_{a}^{b} f(t) d t$ exist.

Received February 22, 2016 - Accepted June 02, 2016.

(C)The Author(s) 2016. This article is published with open access by Sidi Mohamed Ben Abdallah University

${ }^{a}$ Department of Mathematics, Faculty of Science and Information Technology, Irbid National University, 2600 Irbid 21110, Jordan.

e-mail: mwomath@gmail.com 
In the same paper [13], the authors have proved the following inequality:

Theorem 1.1. Let $f, u:[a, b] \rightarrow \mathbb{R}$ be such that $u$ is of bounded variation on $[a, b]$ and $f$ is Lipschitzian with the constant $K>0$. Then we have

$$
|\mathcal{D}(f ; u)| \leq \frac{1}{2} K(b-a) \bigvee_{a}^{b}(u) .
$$

The constant $\frac{1}{2}$ is sharp in the sense that it cannot be replaced by a smaller quantity.

In his interesting work [15], Dragomir has obtained the following inequality:

Theorem 1.2. Let $f, u:[a, b] \rightarrow \mathbb{R}$ be such that $u$ is Lipschitzian on $[a, b]$, i.e.,

$$
|u(y)-u(x)| \leq L|x-y|, \forall x, y \in[a, b], \quad(L>0)
$$

and $f$ is Riemann integrable on $[a, b]$.

If $m, M \in \mathbb{R}$, are such that $m \leq f(x) \leq M$, for any $x \in[a, b]$, then the inequality

$$
|\mathcal{D}(f ; u)| \leq \frac{1}{2} L(M-m)(b-a) .
$$

The constant $\frac{1}{2}$ is sharp in the sense that it cannot be replaced by a smaller quantity.

For other recent inequalities of Gruss type, see [2]-[6], [9]-[16] and [18] and the references therein.

1.1. A weighted Dragomir functional. In order to compare the Stieltjes integral mean with the weighted Riemann integral mean, we define the functional $\mathcal{O D}(f, g ; u)$, as follows:

$$
\mathcal{O D}(f, g ; u):=\frac{1}{u(b)-u(a)} \cdot \int_{a}^{b} f(x) d u(x)-\frac{1}{\int_{a}^{b} g(t) d t} \cdot \int_{a}^{b} f(t) g(t) d t
$$

provided that the both integrals exist and $g(t) \neq 0$, for all $t \in[a, b]$.

In particular, as special cases; we are interested in two functionals:

1: The Dragomir-Fedotov functional:

$$
\begin{aligned}
\mathcal{D}(f ; u) & :=\int_{a}^{b} f(x) d u(x)-\frac{u(b)-u(a)}{b-a} \int_{a}^{b} f(t) d t \\
& =[u(b)-u(a)] \cdot \mathcal{O D}(f, 1 ; u) .
\end{aligned}
$$

2: The weighted integral functional:

$$
\begin{aligned}
\mathcal{E}(f, g ; w) & :=\frac{\int_{a}^{b} f(t) w(t) d t}{\int_{a}^{b} w(t) d t}-\frac{\int_{a}^{b} f(t) g(t) d t}{\int_{a}^{b} g(t) d t} \\
& =\frac{1}{\int_{a}^{b} w(t) d t \int_{a}^{b} g(t) d t} \cdot \mathcal{O} \mathcal{D}\left(f, g ; \int_{a}^{x} w(s) d s\right)
\end{aligned}
$$


where, $u(x)=\int_{a}^{x} w(s) d s, w:[a, b] \rightarrow \mathbb{R}$ is continuous on $[a, b]$, and $g(t), w(t) \neq$ 0 , for all $t \in[a, b]$. For more works about this type of integral functional the reader may refer to [1], [7, 8] and [17].

In fact, the functional $\mathcal{O D}(f, g ; u)$ is a natural generalization of Dragomir functional $\mathcal{D}(f ; u)$; so that in this paper, several new bounds with various type of integrators for the functional $\mathcal{O D}(f, g ; u)$ are proved. More specifically, the obtained results deal with integrands of $r$ - $H$-Hölder type, and integrators of bounded variation, Lipschitzian and monotonic types. Through the assumptions for the functions involved in the functionals (5) and (6) several bounds may be obtained by a direct substitution and we shall omit the details.

\section{The case of bounded variation integrators}

The following result holds:

Theorem 2.1. Let $f, u, g:[a, b] \rightarrow \mathbb{R}$ be mappings such that $f$ is of $r$-H-Hölder type on $[a, b]$, where $r \in(0,1]$ and $H>0$ are given, and $u$ is of bounded variation on $[a, b]$. Then we have the inequality:

$$
\leq \frac{|\mathcal{O D}(f, g ; u)|}{[u(b)-u(a)] \cdot \int_{a}^{b} g(t) d t} \begin{cases}\frac{(b-a)^{r+1}}{r+1} \cdot\|g\|_{\infty} \cdot \bigvee_{a}^{b}(u), & \text { if } \quad g \in L_{\infty}[a, b], \\ \frac{(b-a)^{(q r+1) / q}}{(q r+1)^{1 / q}} \cdot\|g\|_{p} \cdot \bigvee_{a}^{b}(u), & \text { if } \quad g \in L_{p}[a, b], \\ & p>1, \frac{1}{p}+\frac{1}{q}=1, \\ (b-a)^{r}\|g\|_{1} \cdot \bigvee_{a}^{b}(u), & \text { if } g \in L_{1}[a, b] .\end{cases}
$$

where, $\bigvee_{a}^{b}(u)$ is the total variation of $u$ over $[a, b]$.

Proof. It is well-known that for a continuous function $p:[a, b] \rightarrow \mathbb{R}$ and a function $\nu:[a, b] \rightarrow \mathbb{R}$ of bounded variation, one has the inequality

$$
\left|\int_{a}^{b} p(t) d \nu(t)\right| \leq \sup _{t \in[a, b]}|p(t)| \bigvee_{a}^{b}(\nu) .
$$

Therefore, as $u$ is of bounded variation on $[a, b]$, we have

$$
\begin{aligned}
& {[u(b)-u(a)] \cdot\left(\int_{a}^{b} g(t) d t\right) \cdot|\mathcal{O D}(f, g ; u)|} \\
& =\left|\int_{a}^{b}\left[f(x) \int_{a}^{b} g(t) d t-\int_{a}^{b} f(t) g(t) d t\right] d u(x)\right| \\
& \leq \sup _{x \in[a, b]}\left|\int_{a}^{b}[f(x)-f(t)] g(t) d t\right| \cdot \bigvee_{a}^{b}(u) .
\end{aligned}
$$


As $f$ is of $r$-H-Hölder type on $[a, b]$ and $g \in L_{\infty}[a, b]$, then we have

$$
\begin{aligned}
\left|\int_{a}^{b}[f(x)-f(t)] g(t) d t\right| & \leq \int_{a}^{b}|f(x)-f(t)||g(t)| d t \\
& \leq H \int_{a}^{b}|x-t|^{r}|g(t)| d t \\
& \leq H \sup _{t \in[a, b]}|g(t)| \cdot \int_{a}^{b}|x-t|^{r} d t \\
& =\frac{H}{r+1}\left[(x-a)^{r+1}+(b-x)^{r+1}\right] \cdot\|g\|_{\infty} .
\end{aligned}
$$

It follows that

$$
\sup _{x \in[a, b]}\left|\int_{a}^{b}[f(x)-f(t)] g(t)\right| d x \leq \frac{H}{r+1}(b-a)^{r+1} \cdot\|g\|_{\infty} .
$$

Combining (8) and (10), we get the first inequality in (7).

To prove the second inequality in (7). As $f$ is of $r$-H-Hölder type on $[a, b]$, then we have

$$
\begin{aligned}
\left|\int_{a}^{b}[f(x)-f(t)] g(t) d t\right| & \leq \int_{a}^{b}|f(x)-f(t)||g(t)| d t \\
& \leq H \int_{a}^{b}|x-t|^{r}|g(t)| d t .
\end{aligned}
$$

Now, as $g \in L_{p}[a, b]$ therefore, by applying the well-known Hölder integral inequality, we get

$$
\begin{aligned}
\left|\int_{a}^{b}[f(x)-f(t)] g(t) d t\right| & \leq H \int_{a}^{b}|x-t|^{r}|g(t)| d t \\
& \leq H\left(\int_{a}^{b}|x-t|^{r q} d t\right)^{1 / q}\left(\int_{a}^{b}|g(t)|^{p} d t\right)^{1 / p} \\
& =\frac{H}{(q r+1)^{1 / q}}\left[(x-a)^{q r+1}+(b-x)^{q r+1}\right]^{1 / q} \cdot\|g\|_{p} .
\end{aligned}
$$

It follows that

$$
\begin{aligned}
\sup _{x \in[a, b]}\left|\int_{a}^{b}[f(x)-f(t)] g(t) d t\right| & \leq \frac{H}{(q r+1)^{1 / q}} \cdot\|g\|_{p} \cdot \sup _{x \in[a, b]}\left[(x-a)^{q r+1}+(b-x)^{q r+1}\right]^{1 / q} \\
& \leq H \frac{(b-a)^{(q r+1) / q}}{(q r+1)^{1 / q}} \cdot\|g\|_{p} .
\end{aligned}
$$

Combining (8) and (11), we get the second inequality in (7).

Finally, to prove the third inequality in (7). By assumptions we have:

$$
\left|\int_{a}^{b}[f(x)-f(t)] g(t) d t\right| \leq \int_{a}^{b}|f(x)-f(t)||g(t)| d t
$$




$$
\begin{aligned}
& \leq H \int_{a}^{b}|x-t|^{r}|g(t)| d t \\
& \leq H \sup _{t \in[a, b]}\left\{|x-t|^{r}\right\} \int_{a}^{b}|g(t)| d t \\
& =H\|g\|_{1} \max _{t \in[a, b]}\left\{(x-a)^{r},(b-x)^{r}\right\} \\
& =H\|g\|_{1}\left[\max _{t \in[a, b]}\{(x-a),(b-x)\}\right]^{r} \\
& =H\|g\|_{1}\left[\frac{b-a}{2}+\left|x-\frac{a+b}{2}\right|\right]^{r} \\
& \leq H\|g\|_{1}(b-a)^{r} .
\end{aligned}
$$

Combining (8) and (12), we get the third inequality in (7) and thus the theorem is proved.

Corollary 2.1. Let $u$ as in Theorem 2.1 and $f:[a, b] \rightarrow \mathbb{R}$ be an L-Lipschitzian mapping on $[a, b]$. Then we have the inequality

$$
\begin{aligned}
& |\mathcal{O D}(f, g ; u)| \\
& \leq \frac{L}{[u(b)-u(a)] \cdot \int_{a}^{b} g(t) d t}\left\{\begin{array}{ll}
\frac{(b-a)^{2}}{2} \cdot\|g\|_{\infty} \cdot \bigvee_{a}^{b}(u), & \text { if } g \in L_{\infty}[a, b], \\
\frac{(b-a)^{(q+1) / q}}{(q+1)^{1 / q}} \cdot\|g\|_{p} \cdot \bigvee_{a}^{b}(u), & \text { if } \quad g \in L_{p}[a, b], \\
& p>1, \frac{1}{p}+\frac{1}{q}=1, \\
(b-a)\|g\|_{1} \cdot \bigvee_{a}^{b}(u), & \text { if } g \in L_{1}[a, b] .
\end{array} .\right.
\end{aligned}
$$

Corollary 2.2. Assume $f$ as in Theorem 2.1. Let $u \in C^{(1)}[a, b]$. Then we have the inequality

$$
\begin{aligned}
& |\mathcal{O D}(f, g ; u)| \\
& \leq \frac{H}{[u(b)-u(a)] \cdot \int_{a}^{b} g(t) d t}\left\{\begin{array}{lc}
\frac{(b-a)^{r+1}}{r+1} \cdot\|g\|_{\infty} \cdot\left\|u^{\prime}\right\|_{1,[a, b]}, & \text { if } \quad g \in L_{\infty}[a, b], \\
\frac{(b-a)^{(q r+1) / q}}{(q r+1)^{1 / q}} \cdot\|g\|_{p} \cdot\left\|u^{\prime}\right\|_{1,[a, b]}, & \text { if } \quad g \in L_{p}[a, b], \\
& p>1, \frac{1}{p}+\frac{1}{q}=1, \\
(b-a)^{r}\|g\|_{1} \cdot\left\|u^{\prime}\right\|_{1,[a, b]}, & \text { if } \quad g \in L_{1}[a, b] .
\end{array},\right.
\end{aligned}
$$

where $\|\cdot\|_{1}$ is the $L_{1}$ norm, namely $\left\|u^{\prime}\right\|_{1,[a, b]}:=\int_{a}^{b}\left|u^{\prime}(t)\right| d t$.

Corollary 2.3. Assume $f$ as in Theorem 2.1. Let $u:[a, b] \rightarrow \mathbb{R}$ be a K-Lipschitzian mapping with the constant $K>0$. Then we have the inequality

$$
|\mathcal{O D}(f, g ; u)|
$$




$$
\leq \frac{H K(b-a)}{[u(b)-u(a)] \cdot \int_{a}^{b} g(t) d t}\left\{\begin{array}{ll}
\frac{(b-a)^{r+1}}{r+1} \cdot\|g\|_{\infty}, & \text { if } \quad g \in L_{\infty}[a, b], \\
\frac{(b-a)^{(q r+1) / q}}{(q r+1)^{1 / q}} \cdot\|g\|_{p}, & \text { if } \quad g \in L_{p}[a, b], \\
& p>1, \frac{1}{p}+\frac{1}{q}=1, \\
(b-a)^{r}\|g\|_{1}, & \text { if } \quad g \in L_{1}[a, b] .
\end{array} .\right.
$$

Corollary 2.4. Assume $f$ as in Theorem 2.1. Let $u:[a, b] \rightarrow \mathbb{R}$ be a monotonic increasing mapping. Then we have the inequality

$$
\begin{aligned}
& |\mathcal{O D}(f, g ; u)| \\
& \leq \frac{H}{\int_{a}^{b} g(t) d t}\left\{\begin{array}{ll}
\frac{(b-a)^{r+1}}{r+1} \cdot\|g\|_{\infty}, & \text { if } \quad g \in L_{\infty}[a, b], \\
\frac{(b-a)^{(q r+1) / q}}{(q r+1)^{1 / q}} \cdot\|g\|_{p}, & \text { if } \quad g \in L_{p}[a, b], \\
& p>1, \frac{1}{p}+\frac{1}{q}=1, \\
(b-a)^{r}\|g\|_{1}, & \text { if } g \in L_{1}[a, b] .
\end{array} .\right.
\end{aligned}
$$

Remark 2.1. For the last three inequalities, one may deduce several inequalities for L-Lipschitzian integrands by setting $r=1$ and replace $H$ by $L$. We left the details to the interested reader.

Remark 2.2. Under the assumptions of Theorem 2.1, one may deduce several inequalities for the Dragomir-Fedotov functional (5) and the weighted integral functional (6).

\section{The case of Lipschitzian integrators}

Theorem 3.1. Let $f:[a, b] \rightarrow \mathbb{R}$ be an $r$-H-Hölder type mapping on $[a, b]$, and $u:[a, b] \rightarrow \mathbb{R}$ be an L-Lipschitzian mapping on $[a, b]$, where $r$ and $H, L>0$ are given. Then we have the inequality

$$
\begin{aligned}
& |\mathcal{O D}(f, g ; u)| \\
& \leq \frac{L H}{[u(b)-u(a)] \cdot \int_{a}^{b} g(t) d t}\left\{\begin{array}{rr}
\frac{2(b-a)^{r+2}}{(r+1)(r+2)} \cdot\|g\|_{\infty}, & \text { if } g \in L_{\infty}[a, b], \\
\frac{2 q}{(q r+1)^{1 / q}} \cdot \frac{(b-a)^{(q(r+1)+1) / q}}{(q(r+1)+1)} \cdot\|g\|_{p}, & \text { if } \quad g \in L_{p}[a, b], \\
p>1, \frac{1}{p}+\frac{1}{q}=1, & \\
\frac{\left(2^{r+1}-1\right)}{2^{r}(r+1)}(b-a)^{r+1}\|g\|_{1}, & \text { if } g \in L_{1}[a, b] .
\end{array} .\right.
\end{aligned}
$$


Proof. It is well-known that for a Riemann integrable function $p:[a, b] \rightarrow \mathbb{R}$ and $L$-Lipschitzian function $\nu:[a, b] \rightarrow \mathbb{R}$, one has the inequality

$$
\left|\int_{a}^{b} p(t) d \nu(t)\right| \leq L \int_{a}^{b}|p(t)| d t
$$

Therefore, as $u$ is $L$-Lipschitzian on $[a, b]$, we have

$$
\begin{aligned}
& {[u(b)-u(a)] \cdot\left(\int_{a}^{b} g(t) d t\right) \cdot|\mathcal{O D}(f, g ; u)|} \\
& =\left|\int_{a}^{b}\left[f(x) \int_{a}^{b} g(t) d t-\int_{a}^{b} f(t) g(t) d t\right] d u(x)\right| \\
& \leq L \int_{a}^{b}\left|\int_{a}^{b}[f(x)-f(t)] g(t) d t\right| d x .
\end{aligned}
$$

As $f$ is of $r$-H-Hölder type on $[a, b]$ and $g \in L_{\infty}[a, b]$, by (9) we have

$$
\left|\int_{a}^{b}[f(x)-f(t)] g(t) d t\right| \leq \frac{H}{r+1}\left[(x-a)^{r+1}+(b-x)^{r+1}\right] \cdot\|g\|_{\infty} .
$$

It follows that

$$
\begin{aligned}
\int_{a}^{b}\left|\int_{a}^{b}[f(x)-f(t)] g(t) d t\right| d x & \leq \frac{H}{r+1} \cdot\|g\|_{\infty} \cdot \int_{a}^{b}\left[(x-a)^{r+1}+(b-x)^{r+1}\right] d x \\
& \leq \frac{2 H}{(r+1)(r+2)}(b-a)^{r+2} \cdot\|g\|_{\infty}
\end{aligned}
$$

Combining (18) and (20), we get the first inequality in (17).

To prove the second inequality in (17). As $f$ is of $r$-H-Hölder type on $[a, b]$, then we have

$$
\begin{aligned}
\left|\int_{a}^{b}[f(x)-f(t)] g(t) d t\right| & \leq \int_{a}^{b}|f(x)-f(t)||g(t)| d t \\
& \leq H \int_{a}^{b}|x-t|^{r}|g(t)| d t
\end{aligned}
$$

Now, as $g \in L_{p}[a, b]$ therefore, by applying the well-known Hölder integral inequality, we get

$$
\begin{aligned}
\left|\int_{a}^{b}[f(x)-f(t)] g(t) d t\right| & \leq H \int_{a}^{b}|x-t|^{r}|g(t)| d t \\
& \leq H\left(\int_{a}^{b}|x-t|^{r q} d t\right)^{1 / q}\left(\int_{a}^{b}|g(t)|^{p} d t\right)^{1 / p} \\
& =\frac{H}{(q r+1)^{1 / q}}\left[(x-a)^{q r+1}+(b-x)^{q r+1}\right]^{1 / q} \cdot\|g\|_{p}
\end{aligned}
$$




$$
=\frac{H}{(q r+1)^{1 / q}}\left[\left((x-a)^{\left(r+\frac{1}{q}\right)}\right)^{q}+\left((b-x)^{\left(r+\frac{1}{q}\right)}\right)^{q}\right]^{1 / q} \cdot\|g\|_{p} .
$$

Using the fact that $\left(A^{s}+B^{s}\right)^{1 / s} \leq(A+B)$, for all $A, B \geq 0$ and $s \geq 1$, it follows that

$$
\begin{aligned}
& \int_{a}^{b}\left|\int_{a}^{b}[f(x)-f(t)] g(t) d t\right| d x \\
& \leq \frac{H}{(q r+1)^{1 / q}} \cdot\|g\|_{p} \int_{a}^{b}\left[((x-a))^{\left(r+\frac{1}{q}\right)}\right)^{q}+((b-x) \\
& \left.\leq \frac{H}{(q r+1)^{1 / q}} \cdot\|g\|_{p} \cdot \int_{a}^{b}[(x-a))^{q}\right]^{\left(r+\frac{1}{q}\right)}+(b-x) \\
& \left.\left.\leq r+\frac{1}{q}\right)\right] d x \\
& \leq H \frac{2 q}{(q r+1)^{1 / q}} \cdot \frac{(b-a)^{(q(r+1)+1) / q}}{(q(r+1)+1)} \cdot\|g\|_{p} .
\end{aligned}
$$

Combining (18) and (22), we get the second inequality in (17).

Finally, to prove the third inequality in (17). By assumptions we have:

$$
\left|\int_{a}^{b}[f(x)-f(t)] g(t) d t\right| \leq H\|g\|_{1}\left[\frac{b-a}{2}+\left|x-\frac{a+b}{2}\right|\right]^{r}
$$

It follows that

$$
\begin{aligned}
\int_{a}^{b}\left|\int_{a}^{b}[f(x)-f(t)] g(t) d t\right| d x & \leq H\|g\|_{1} \cdot \int_{a}^{b}\left[\frac{b-a}{2}+\left|x-\frac{a+b}{2}\right|\right]^{r} d x \\
& =H\|g\|_{1} \frac{\left(2^{r+1}-1\right)}{2^{r}(r+1)}(b-a)^{r+1} .
\end{aligned}
$$

Combining (18) and (24), we get the third inequality in (17) and thus the theorem is proved.

Corollary 3.1. Let $u$ as in Theorem 3.1 and $f:[a, b] \rightarrow \mathbb{R}$ be an $K$-Lipschitzian mapping on $[a, b]$. Then we have the inequality

$|\mathcal{O D}(f, g ; u)|$

$$
\leq \frac{L K}{[u(b)-u(a)] \cdot \int_{a}^{b} g(t) d t}\left\{\begin{array}{ll}
\frac{(b-a)^{3}}{3} \cdot\|g\|_{\infty}, & \text { if } g \in L_{\infty}[a, b], \\
\frac{2 q}{(q+1)^{1 / q}} \cdot \frac{(b-a)^{(2 q+1) / q}}{(2 q+1)} \cdot\|g\|_{p}, & \text { if } g \in L_{p}[a, b], \\
\frac{3}{4}(b-a)^{2}\|g\|_{1}, & \text { if } g \in 1, \frac{1}{p}+\frac{1}{q}=1,
\end{array} .\right.
$$

Remark 3.1. Under the assumptions of Theorem 3.1, one may deduce several inequalities for the functionals (5) and (6). 


\section{The case of monotonic integrators}

Theorem 4.1. Let $f:[a, b] \rightarrow \mathbb{R}$ be an $r$-H-Hölder type mapping on $[a, b]$, and $u:[a, b] \rightarrow \mathbb{R}$ be a monotonic mapping on $[a, b]$, where $r$ and $H>0$ are given. Then we have the inequality

$$
\begin{aligned}
& |\mathcal{O D}(f, g ; u)| \\
& \leq \frac{H}{[u(b)-u(a)] \cdot \int_{a}^{b} g(t) d t}\left\{\begin{array}{ll}
\frac{2 \frac{(b-a)^{r+1}}{r+1} \cdot\|g\|_{\infty} \cdot[u(b)-u(a)],}{} & \text { if } g \in L_{\infty}[a, b], \\
\frac{2(b-a)^{r+\frac{1}{q}}}{(q r+1)^{1 / q}} \cdot\|g\|_{p} \cdot[u(b)-u(a)], & \text { if } g \in L_{p}[a, b] ; \\
(b-a)^{r}\|g\|_{1} \cdot[u(b)-u(a)], & p>1, \frac{1}{p}+\frac{1}{q}=1,
\end{array} .\right.
\end{aligned}
$$

Proof. It is well-known that for a monotonic non-decreasing function $\nu:[a, b] \rightarrow \mathbb{R}$ and continuous function $p:[a, b] \rightarrow \mathbb{R}$, one has the inequality

$$
\left|\int_{a}^{b} p(t) d \nu(t)\right| \leq \int_{a}^{b}|p(t)| d \nu(t)
$$

Therefore, as $u$ is monotonic non-decreasing on $[a, b]$, we have

$$
\begin{aligned}
& {[u(b)-u(a)] \cdot\left(\int_{a}^{b} g(t) d t\right) \cdot|\mathcal{O D}(f, g ; u)|} \\
& =\frac{1}{(b-a)^{2}}\left|\int_{a}^{b}\left[f(x) \int_{a}^{b} g(t) d t-\int_{a}^{b} f(t) g(t) d t\right] d u(x)\right| \\
& \leq \int_{a}^{b}\left|f(x) \int_{a}^{b} g(t) d t-\int_{a}^{b} f(t) g(t) d t\right| d u(x) \\
& =\int_{a}^{b}\left|\int_{a}^{b}[f(x)-f(t)] g(t) d t\right| d u(x) .
\end{aligned}
$$

As $f$ is of $r$-H-Hölder type on $[a, b]$ and $g \in L_{\infty}[a, b]$, by (9) we have

$$
\left|\int_{a}^{b}[f(x)-f(t)] g(t) d t\right| \leq \frac{H}{r+1}\left[(x-a)^{r+1}+(b-x)^{r+1}\right] \cdot\|g\|_{\infty} .
$$

It follows that

$$
\begin{aligned}
\int_{a}^{b} \mid \int_{a}^{b}[f(x)-f(t)] g(t) & d t \mid d u(x) \\
\leq & \frac{H}{r+1} \cdot\|g\|_{\infty} \cdot \int_{a}^{b}\left[(x-a)^{r+1}+(b-x)^{r+1}\right] d u(x) .
\end{aligned}
$$

Now, using Riemann-Stieltjes integral we have

$$
\int_{a}^{b}(x-a)^{r+1} d u(x)=(b-a)^{r+1} u(b)-(r+1) \int_{a}^{b}(x-a)^{r} u(x) d x
$$


and

$$
\int_{a}^{b}(b-x)^{r+1} d u(x)=-(b-a)^{r+1} u(a)+(r+1) \int_{a}^{b}(b-x)^{r} u(x) d x .
$$

Adding (30) and (31), we get

$$
\begin{aligned}
& \int_{a}^{b}\left[(x-a)^{r+1}+(b-x)^{r+1}\right] d u(x) . \\
& \quad=(b-a)^{r+1}[u(b)-u(a)]+(r+1) \int_{a}^{b}\left[(b-x)^{r}-(x-a)^{r}\right] u(x) d x .
\end{aligned}
$$

Now, by the monotonicity property of $u$ we have

$$
\int_{a}^{b}(x-a)^{r} u(x) d x \geq u(a) \int_{a}^{b}(x-a)^{r} d x=\frac{(b-a)^{r+1}}{r+1} \cdot u(a),
$$

and

$$
\int_{a}^{b}(b-x)^{r} u(x) d x \leq u(b) \int_{a}^{b}(b-x)^{r} d x=\frac{(b-a)^{r+1}}{r+1} \cdot u(b) .
$$

Substituting (33) and (34) in (32), we get

$$
\int_{a}^{b}\left[(x-a)^{r+1}+(b-x)^{r+1}\right] d u(x) \leq 2(b-a)^{r+1}[u(b)-u(a)] .
$$

Substituting (35) in (29), we get

$$
\int_{a}^{b}\left|\int_{a}^{b}[f(x)-f(t)] g(t) d t\right| d u(x) \leq 2 H \frac{(b-a)^{r+1}}{r+1} \cdot\|g\|_{\infty} \cdot[u(b)-u(a)],
$$

and therefore, by (27) we get the first inequality in (26).

To prove the second inequality in (26). As $f$ is of $r$-H-Hölder type on $[a, b]$ and $g \in L_{p}[a, b]$ therefore, by (21), we have

$$
\begin{aligned}
& \left|\int_{a}^{b}[f(x)-f(t)] g(t) d t\right| \\
& \leq \frac{H}{(q r+1)^{1 / q}}\left[\left((x-a)^{\left(r+\frac{1}{q}\right)}\right)^{q}+\left((b-x)^{\left(r+\frac{1}{q}\right)}\right)^{q}\right]^{1 / q} \cdot\|g\|_{p} .
\end{aligned}
$$

It follows by (22), that

$$
\begin{aligned}
& \int_{a}^{b}\left|\int_{a}^{b}[f(x)-f(t)] g(t) d t\right| d u(x) \\
& \leq \frac{H}{(q r+1)^{1 / q}} \cdot\|g\|_{p} \int_{a}^{b}\left[\left((x-a)^{\left(r+\frac{1}{q}\right)}\right)^{q}+\left((b-x)^{\left(r+\frac{1}{q}\right)}\right)^{q}\right]^{1 / q} d u(x) \\
& \leq \frac{H}{(q r+1)^{1 / q}} \cdot\|g\|_{p} \cdot \int_{a}^{b}\left[(x-a)^{\left(r+\frac{1}{q}\right)}+(b-x)^{\left(r+\frac{1}{q}\right)}\right] d u(x) .
\end{aligned}
$$


Now, using Riemann-Stieltjes integral we have

$$
\begin{aligned}
\int_{a}^{b}(x-a)^{r+\frac{1}{q}} d u(x) & \\
& =(b-a)^{r+\frac{1}{q}} u(b)-\left(r+\frac{1}{q}\right) \int_{a}^{b}(x-a)^{r+\frac{1}{q}-1} u(x) d x,
\end{aligned}
$$

and

$$
\begin{aligned}
& \int_{a}^{b}(b-x)^{r+\frac{1}{q}} d u(x) \\
&=-(b-a)^{r+\frac{1}{q}} u(a)+\left(r+\frac{1}{q}\right) \int_{a}^{b}(b-x)^{r+\frac{1}{q}-1} u(x) d x .
\end{aligned}
$$

Adding (38) and (39), we get

$$
\begin{aligned}
& \int_{a}^{b}\left[(x-a)^{r+1}+(b-x)^{r+1}\right] d u(x) . \\
& =(b-a)^{r+\frac{1}{q}}[u(b)-u(a)]+\left(r+\frac{1}{q}\right) \int_{a}^{b}\left[(b-x)^{r+\frac{1}{q}-1}-(x-a)^{r+\frac{1}{q}-1}\right] u(x) d x .
\end{aligned}
$$

Now, by the monotonicity property of $u$ we have

$$
\begin{aligned}
\int_{a}^{b}(x-a)^{r+\frac{1}{q}-1} u(x) d x & \geq u(a) \int_{a}^{b}(x-a)^{r+\frac{1}{q}-1} d x \\
& =\frac{q}{q r+1}(b-a)^{r+\frac{1}{q}} \cdot u(a)
\end{aligned}
$$

and

$$
\begin{aligned}
\int_{a}^{b}(b-x)^{r+\frac{1}{q}-1} u(x) d x & \leq u(b) \int_{a}^{b}(b-x)^{r+\frac{1}{q}-1} d x \\
& =\frac{q}{q r+1}(b-a)^{r+\frac{1}{q}} \cdot u(b) .
\end{aligned}
$$

Substituting (41) and (42) in (40), we get

$$
\int_{a}^{b}\left[(x-a)^{r+1}+(b-x)^{r+1}\right] d u(x) \leq 2(b-a)^{r+\frac{1}{q}}[u(b)-u(a)] .
$$

Substituting (43) in (37), we get

$$
\begin{aligned}
\int_{a}^{b}\left|\int_{a}^{b}[f(x)-f(t)] g(t) d t\right| d u(x) & \\
\leq & \frac{2 H}{(q r+1)^{1 / q}}(b-a)^{r+\frac{1}{q}} \cdot\|g\|_{p} \cdot[u(b)-u(a)],
\end{aligned}
$$

and therefore, by (27) we get the second inequality in (26). 
Finally, to prove the third inequality in (26). As $f$ is of $r$-H-Hölder type on $[a, b]$ and $g \in L_{1}[a, b]$ therefore, by (23), we have

$$
\left|\int_{a}^{b}[f(x)-f(t)] g(t) d t\right| \leq H\|g\|_{1}\left[\frac{b-a}{2}+\left|x-\frac{a+b}{2}\right|\right]^{r},
$$

which gives by (27), that

$$
\begin{aligned}
\int_{a}^{b}\left|\int_{a}^{b}[f(x)-f(t)] g(t) d t\right| d u & (x) \\
& \leq H\|g\|_{1} \cdot \int_{a}^{b}\left[\frac{b-a}{2}+\left|x-\frac{a+b}{2}\right|\right]^{r} d u(x) .
\end{aligned}
$$

Now, using Riemann-Stieltjes integral we have

$$
\begin{aligned}
& \int_{a}^{b}\left[\frac{b-a}{2}+\left|x-\frac{a+b}{2}\right|\right]^{r} d u(x) \\
& =\int_{a}^{\frac{a+b}{2}}(b-x)^{r} d u(x)+\int_{\frac{a+b}{2}}^{b}(x-a)^{r} d u(x) \\
& =\left(\frac{b-a}{2}\right)^{r} u\left(\frac{a+b}{2}\right)-(b-a)^{r} u(a)+r \int_{a}^{\frac{a+b}{2}}(b-x)^{r-1} u(x) d x \\
& \quad+(b-a)^{r} u(b)-\left(\frac{b-a}{2}\right)^{r} u\left(\frac{a+b}{2}\right)-r \int_{\frac{a+b}{2}}^{b}(x-a)^{r-1} u(x) d x \\
& =(b-a)^{r}[u(b)-u(a)]+r\left[\int_{a}^{\frac{a+b}{2}}(b-x)^{r-1} u(x) d x-\int_{\frac{a+b}{2}}^{b}(x-a)^{r-1} u(x) d x\right] .
\end{aligned}
$$

Now, by the monotonicity property of $u$ we have

$$
\begin{aligned}
\int_{a}^{\frac{a+b}{2}}(b-x)^{r-1} u(x) d x & \leq u\left(\frac{a+b}{2}\right) \int_{a}^{\frac{a+b}{2}}(b-x)^{r-1} d x \\
& =\frac{\left(2^{r}-1\right)}{r 2^{r}}(b-a)^{r} \cdot u\left(\frac{a+b}{2}\right)
\end{aligned}
$$

and

$$
\begin{aligned}
\int_{\frac{a+b}{2}}^{b}(x-a)^{r-1} u(x) d x & \geq u\left(\frac{a+b}{2}\right) \int_{\frac{a+b}{2}}^{b}(x-a)^{r-1} d x \\
& =\frac{\left(2^{r}-1\right)}{r 2^{r}}(b-a)^{r} \cdot u\left(\frac{a+b}{2}\right)
\end{aligned}
$$


Substituting (47) and (48) in (46), we get

$$
\int_{a}^{b}\left[\frac{b-a}{2}+\left|x-\frac{a+b}{2}\right|\right]^{r} d u(x) \leq(b-a)^{r}[u(b)-u(a)] .
$$

Substituting (49) in (45), we get

$$
\int_{a}^{b}\left|\int_{a}^{b}[f(x)-f(t)] g(t) d t\right| d u(x) \leq H\|g\|_{1} \cdot(b-a)^{r}[u(b)-u(a)] .
$$

and therefore, by (27) we get the third inequality in (26), and thus the theorem is proved.

Remark 4.1. Under the assumptions of Theorem 4.1, one may deduce several inequalities for the functionals (5) and (6).

\section{References}

[1] M.W. Alomari, Difference between two Stieltjes integral means, Kragujevac Journal of Mathematics, 38(1) (2014), 35-49.

[2] M.W. Alomari, New Grüss type inequalities for double integrals, Appl. Math. Comp., 228 (2014) 102-107.

[3] M.W. Alomari and S.S. Dragomir, New Grüss type inequalities for Riemann-Stieltjes integral with monotonic integrators and applications, Ann. Funct. Anal., 5 (2014), no. 1, 77-93.

[4] M.W. Alomari and S.S. Dragomir, Mercer-Trapezoid rule for Riemann-Stieltjes integral with applications, Journal of Advances in Mathematics, 2 (2) (2013), 67-85.

[5] N.S. Barnett, S.S. Dragomir and I. Gomma, A companion for the Ostrowski and the generalised trapezoid inequalities, Mathematical and Computer Modelling, 50 (2009), 179-187.

[6] N.S. Barnett, W.-S. Cheung, S.S. Dragomir, A. Sofo, Ostrowski and trapezoid type inequalities for the Stieltjes integral with Lipschitzian integrands or integrators, Comp. Math. Appl. , 57 (2009), 195-201.

[7] N.S. Barnett, P. Cerone, S.S. Dragomir and A.M. Fink, Comparing two integral means for absolutely continuous mappings whose derivatives are in $L_{\infty}[a, b]$ and applications, Comp. and Math. Appl., 44 (1/2) (2002), 241-251.

[8] P. Cerone and S.S. Dragomir, Differences between means with bounds from a Riemann-Stieltjes integral, Comp. Math. Appl., 46 (2003) 445-453.

[9] P. Cerone, W.S. Cheung, S.S. Dragomir, On Ostrowski type inequalities for Stieltjes integrals with absolutely continuous integrands and integrators of bounded variation, Comp. Math. Appl., 54 (2007), 183-191.

[10] P. Cerone, S.S. Dragomir, New bounds for the three-point rule involving the Riemann-Stieltjes integrals, in: C. Gulati, et al. (Eds.), Advances in Statistics Combinatorics and Related Areas, World Science Publishing, 2002, pp. 53-62. 
[11] P. Cerone, S.S. Dragomir, Approximating the Riemann-Stieltjes integral via some moments of the integrand, Mathematical and Computer Modelling, 49 (2009), 242-248.

[12] S.S. Dragomir and Th.M. Rassias (Ed.), Ostrowski Type Inequalities and Applications in Numerical Integration, Kluwer Academic Publishers, Dordrecht, 2002.

[13] S.S. Dragomir, I. Fedotov, An inequality of Grüss type for Riemann-Stieltjes integral and applications for special means, Tamkang J. Math., 29 (4) (1998) 287-292

[14] S.S. Dragomir, I. Fedotov, A Grüss type inequality for mappings of bounded variation and applications to numerical analysis, Nonlinear Funct. Anal. Appl., 6 (3) (2001) 425-433.

[15] S.S. Dragomir, Inequalities of Grüss type for the Stieltjes integral and applications, Kragujevac J. Math., 26 (2004) 89-112.

[16] S.S. Dragomir, C. Buşe, M.V. Boldea, L. Braescu, A generalisation of the trapezoid rule for the Riemann-Stieltjes integral and applications, Nonlinear Anal. Forum 6 (2) (2001) 33-351.

[17] A.I. Kechriniotis and N.D. Assimakis, On the inequality of the difference of two integral means and applications for pdfs, J. Ineq. pure Appl. math., 8 (1) (2007), Article 10, 6 pp.

[18] Z. Liu, Refinement of an inequality of Grüss type for Riemann-Stieltjes integral, Soochow J. Math., 30 (4) (2004) 483-489. 